\title{
Management of Cauda Equina Syndrome: NHS Staff Perceptions in a DGH
}

\section{Taylor $\mathbf{G}^{1 *}$, Tanagho $\mathrm{A}^{2}$ and Rutherford $\mathrm{M}^{3}$}

${ }^{1}$ Advanced Practitioner Physiotherapist in Emergency Department and Orthopaedics, University Hospital, Crosshouse, UK

${ }^{2}$ Consultant Orthopaedic Surgeon in Trauma \& Orthopaedics, University Hospital Crosshouse, UK

${ }^{3}$ Lecturer, Department of Physiotherapy and Paramedicne, Glasgow Caledonian University, UK

\section{Research Article}

Volume 4 Issue 2

Received Date: September 30, 2020

Published Date: October 14, 2020

DOI: $10.23880 /$ jobd-16000199

*Corresponding author: G Taylor, Advanced Practitioner Physiotherapist in Emergency Department and Orthopaedics, University Hospital Crosshouse, Kilmarnock Road, KA2 0BE, Scotland, UK, Tel: +441563521133; Email: Gemma.Taylor2@aapct. scot.nhs.uk

\section{Abstract}

Objective: Cauda Equina Syndrome (CES) is a challenging condition to diagnose due to such variability in clinical features and poor correlation with MRI findings. It requires surgery within hours to avoid damage to the bowel, bladder, sexual organs and lower limbs. There are empirical studies exploring patients' perspectives of having CES, however, there is no literature to review NHS staff perceptions of managing this neurosurgical emergency. The aim is to explore perceptions of senior clinical staff who commonly manage patients with suspected CES in secondary care within the Emergency Department (ED) and Trauma and Orthopaedics (T\&O) to improve the management of CES. The main objective is to identify barriers to successful management and possible solutions to improve care.

Methods: A self-administered non-validated questionnaire was designed to identify barriers experienced and potential solutions. In addition, a Nominal Group Technique (NGT) was used to gain a greater insight. The qualitative data obtained was analysed by thematic analysis.

Results: The questionnaire generated a 73\% response rate. Six senior clinicians attended the NGT with representation from the three clinical specialities; ED, T\&O and Advanced Physiotherapy Practitioner (APP). The challenges and potential solutions were categorised into themes and subthemes. The barriers highlighted included; staff confidence in clinical diagnosis; fear of litigation and inter-professional relations; patient psychosocial factors, expectations and comorbidities; lack of agreed local pathway. The solutions raised include; staff training; standardising local/national pathway; access to daily Magnetic Resonance Imaging (MRI) slots.

Conclusion: Patient safety and accurate diagnosis of CES is at the forefront of decision-making despite organisation barriers and limitations of existing pathways. A shift away from admission to expedite an urgent MRI requires a change to traditional clinical practice and expectations. In times of rising pressures and financial constraints, collaborative working is essential to implement and sustain the required changes highlighted in this study.

Keywords: Cauda Equina Syndrome; Staff Perceptions 


\section{Journal of Orthopedics \& Bone Disorders}

Abbreviations: CES: Cauda Equina Syndrome; MRI: Magnetic Resonance Imaging; APP: Advanced Physiotherapy Practitioner; NGT: Nominal Group Technique; T \& O: Trauma and Orthopaedics; ED: Emergency Department;

\section{Introduction}

Cauda Equina Syndrome (CES) is a neurosurgical emergency caused by compression of the lumbar and sacral nerve roots resulting in bladder, bowel and sexual dysfunction with potential lower limb weakness and numbness. The most likely cause is a disc herniation, although it is considered rare, accounting for only $2 \%$ of all herniated discs $[1,2]$. However rare, it is highly litigious, which potentially influences clinical decision-making. Failure to act on symptoms of CES has the potential to cause significant morbidity often leading to medicolegal action, with average compensation suggested to be $£ 336,000$ [3]. The drive to detect earlier signs of CES is in response to the impact of residual disability and the significant medicolegal consequences [3].

Accurate identification is essential but regardless of good clinical acumen and level of clinical experience, MRI for primary triage has been encouraged. It is widely acknowledged that there is poor correlation of clinical features with MRI findings with some authors reporting this to vary between 14-48 \%. Urgent access to MRI can be problematic, particularly at local hospitals without 24-hour access to MRI. 24-hour access to MRI is recommended at DGHs and only patients with positive scans being transferred for surgical assessment. In the current financial climate this is not possible due to lack of resource and funding [4].

Early detection is challenging due to such variability in clinical features and high levels of pain. Side effects of medications can also masquerade symptoms. Although the onset of bladder and bowel dysfunction, altered perianal sensation and sexual dysfunction cannot be ignored, they are all multi-factorial in their causes e.g. comorbidities and medication. The challenges in diagnosis may attribute to patient dissatisfaction in care [5].

There have been studies investigating patients' experience of having CES. Such qualitative data encourages clinicians' to reflect on the delivery of care and improve screening of patients at risk.

Studies demonstrated problems caused by patients' lack of understanding of medical terminology and suggest a common, unambiguous language to diagnose CES. Patient centred communication has been linked with positive patient outcomes. Furthermore, effective communication amongst clinicians has been linked with increased staff morale, satisfaction and reduced work stress [6,7]. Patients with suspected CES often interact with different sub-specialities including GPs in primary care, physiotherapy, ED, T\&O and Neurosurgery. It is not unreasonable to consider that this would lead to challenges and inconsistencies in patient care [8].

It would therefore be beneficial to understand clinician's attitudes and beliefs, providing an opportunity to highlight possible challenges and barriers, and if so, propose solutions to improve patient care. Furthermore, exploring the perceptions from different clinical backgrounds is worthwhile to understand if this influences clinical decisions and hopefully identify areas to improve patient centred communication and employ effective team working.

The aim of this research study is to investigate NHS clinical staff perceptions of managing CES. This will include clinicians at a senior level (consultant, registrar or APP) who commonly manage patients with suspected CES in secondary care within ED and T\&O. It aims to gather qualitative data to provide a greater understanding of their experiences in clinical practice and whether or not the limitations mentioned in the literature exist and, if so, what impact does this have on overall management.

The overall aim has been sub-divided using four questions:

- What are the perceptions of managing patients with suspected CES?

- Are there any barriers to successful management of patients with suspected CES?

- What are the solutions to improving overall management of patients with suspected CES?

- Does clinical specialism influence perceptions?

\section{Methodology}

The sampling method used for this qualitative study was purposive sampling, a non-probability sample whereby the participants were selected specifically due to their experience and adequate knowledge of patients with potential CES and therefore the data gathered was relevant and useful.

Inclusion Criteria: Participants included clinical staff working within secondary care in AAH who commonly manage patients with potential CES. The clinical grades and specialities involved included consultants, registrars and APPs from ED and T\&O.

Exclusion Criteria: GPs were excluded as they refer to secondary care for further CES assessment and MRI. Medical staff below registrar level was excluded as they do not make the final decision on clinical outcome. This also helps to 


\section{Journal of Orthopedics \& Bone Disorders}

eliminate level of experience as a contributing factor in staff perceptions.

For the purpose of this study, two research tools were used to gather qualitative data on NHS staff perceptions of managing CES; a self-administered questionnaire and a Nominal Group Technique (NGT). An exploratory mixed method was adopted whereby the information from the questionnaire was used to inform the key aspects to be explored in the NGT.

With careful consideration, a non-validated open-ended self-administered questionnaire was designed as there was no suitable validated alternative, which reduces overall rigour of this study. An open-ended format was used to encourage honest and in-depth opinions. Before dissemination, the questionnaire was piloted by two consultant Allied Health Professionals (AHP's) within Ayrshire. These clinicians were excluded from participating in the main study to eliminate bias. The feedback was acknowledged and relevant changes were made prior to submission for ethical approval.

To encourage participation the questionnaires were disseminated by both email and hard copy. 41 clinicians were invited. Participants were provided with an information leaflet and given 3 weeks for completion. Completion of the questionnaire implied consent.

It was recognised that using this format as the only research tool to obtain qualitative data may be insufficient to produce trustworthy findings. Questionnaires also lack interactive conversation of face-to-face interviews which can provide more in-depth qualitative data. A nominal group technique (NGT) was therefore also conducted to delve beneath superficial responses, aiming to understand the views of staff who regularly contribute to the management of patients with suspected CES. Participants were invited to participate to the NGT via email.

\section{Nominal Group Technique (NGT)}

A nominal group process is a form of qualitative research using structured face-to-face interaction, aiming to research a consensus on a particular topic. Furthermore the NGT is thought to enable decision-making and generate new ideas in healthcare where it may be difficult to compromise. In this particular study, NHS clinical staff from different clinical backgrounds with possible different perspectives could lead to potential disagreement and therefore the NGT was selected over a focus group to minimise a vocal member of staff dominating the conversation [9].
A facilitator was present to ensure organisation throughout the NGT. The facilitator used in this study was a Consultant Physiotherapist who had previous experience of using this technique within the healthcare setting. Participants included two Advanced Physiotherapy Practitioners (APP), three consultants in Emergency Medicine and one Orthopaedic consultant. The author was eliminated from the NGT to reduce researcher bias. The NGT was separated into six sections; overview of the process; silent generation; round robin; clarification; ranking and data management [9].

The two research questions used in the NGT were:

$>$ "From your experience what are the challenges experienced when managing a patient with suspected CES?"

$>$ "What would you suggest to improve overall management of CES?"

Participants were asked to generate and record as many ideas. In the round robin stage, everybody was asked to contribute one point at a time until all points were covered and ideas were discussed. Clarification of ideas enabled further discussion and grouping of the main points raised. Individually, participants were then asked to rank the ideas in level of importance of the possible solutions only ranging from 1-5 and then subsequently the top 3 solutions were agreed amongst the group. It has been acknowledged that one limitation of ranking is the difficulty of eliminating options, as all generated ideas may be deemed important [9]. Due to time constraints, it was agreed that only the potential solutions to the problems identified would be ranked. The NGT session was recorded using an audio recorder and the key points of discussion were scribed. The audiotaped discussions and questionnaires were analysed using a thematic data analysis approach.

\section{Results}

In total, thirty of the forty-one questionnaires were completed resulting in a $73 \%$ response rate, which exceeds the recommendations, by Fincham [10].

The NGT investigated the challenges to managing suspected CES and suggestions for improving patient care.

\section{NGT Results}

Table one presents the main challenges identified, in no particular order of importance, aligned to the clinical specialism that raised the concern. 


\section{Journal of Orthopedics \& Bone Disorders}

\begin{tabular}{|c|c|}
\hline Challenge/Barrier & Relevance to Clinical Specialism \\
\hline Access to timely MRI & ED, T\&O \\
\hline Lack of standardised protocol in Ayrshire and Arran & ED, T\&O and APP \\
\hline No competency framework for clinical assessment & $\mathrm{T} \& 0$ \\
\hline Inconsistent care during OOHs compared with in hours & ED, T\&O \\
\hline Referral from senior ED clinician to junior T\&O medic & ED \\
\hline Overall management is time consuming & ED, T\&O and APP \\
\hline Poor correlation of clinical findings with MRI & ED, T\&O and APP \\
\hline Influence of patient psychosocial factors and other co-morbidities & ED, T\&O and APP \\
\hline Managing patient expectations especially those referred from GPs & ED, T\&O \\
\hline Variability in referrals from GPs & $\mathrm{T} \& 0$ \\
\hline Communication with Neurosurgery & ED, T\&O and APP \\
\hline Time delay to contact Neurosurgery & ED, T\&O and APP \\
\hline $\begin{array}{l}\text { Decide when to MRI: acute onset compared with gradual worsening symptoms. No } \\
\text { evidence to support decision }\end{array}$ & ED, T\&O and APP \\
\hline Fear of litigation & ED, T\&O and APP \\
\hline Fear of missing CES in relation to patient morbidity & ED, T\&O and APP \\
\hline Inconsistent MRI reports & APP \\
\hline Time delay for MRI report & APP \\
\hline Pain management to accurately complete assessment & ED \\
\hline Patients subjected to multiple sensitive examinations in one day & ED and T\&O \\
\hline Feedback on patients referred with suspected CES & APP \\
\hline Atypical presentations and detecting "soft signs" & ED \\
\hline Confirmed CES: wait for transfer to Neurosurgery (can be greater than 10 hours) & T\&O \\
\hline $\begin{array}{l}\text { In-patient referral to T\&O with suspected CES- who's responsibility is it to manage } \\
\text { in-patients in medicine }\end{array}$ & $\mathrm{T} \& 0$ \\
\hline Lack of professional respect when referring patients with suspected CES & APP \\
\hline
\end{tabular}

Table 1: Challenges/Barriers Relating to Clinical Speciality.

Tables 2 \& 3presents the suggested solutions to improving the management of CES agreed by all members of the NGT.

\begin{tabular}{|c|c|}
\hline Proposed Solution & Relevance to Clinical Specialism \\
\hline Standardised national CES pathway & ED, T\&O and APP \\
\hline Standardised pathway in Ayrshire and Arran & ED, T\&O and APP \\
\hline Standardised clinical assessment in Ayrshire and Arran & T\&O \\
\hline Allocated daily MRI slots & ED, T\&O and APP \\
\hline Consistent senior front door point of contact & ED, T\&O \\
\hline Single point of contact (exclude multiple assessment) & ED, T\&O \\
\hline 24 hour access to MRI in Ayrshire and Arran & T\&O \\
\hline CES coordinator: improved liaison for patient referral and transfer & T\&O, APP \\
\hline Improved communication and feedback from Neurosurgery/shared \\
learning & ED, T\&O and APP \\
\hline Improved feedback locally within Ayrshire and Arran & ED \\
\hline Development of care bundle in ED &
\end{tabular}

Table 2: Suggested Improvements for managing CES 


\section{Journal of Orthopedics \& Bone Disorders}

\section{Solutions}

Improved access to MRI

Standardised local CES pathway within Ayrshire and Arran

Improved education of CES in primary and secondary care

Table 3: Top 3 solutions to improve CES management

The narrative data and yielded questionnaire results were analysed thoroughly to produce these two themes:

1. Challenges
2. Solutions

Three further subthemes for each heading were established which are presented in table 4.

\begin{tabular}{|c|c|}
\hline Theme & Sub-theme \\
\hline \multirow{4}{*}{ Challenges } & Staff perceptions and influencing factors \\
\cline { 2 - 2 } & Patient factors and expectations \\
\cline { 2 - 2 } & Lack of standardized pathway and protocol for managing CES \\
\hline \multirow{3}{*}{ Solutions } & MRI access and reporting \\
\cline { 2 - 2 } & Staff education and training \\
\cline { 2 - 2 } & Standardisation CES pathway \\
\hline
\end{tabular}

Table 4: Themes and Subthemes

The sub-themes were further divided (Tables $5 \& 6$ ).

\begin{tabular}{|c|c|}
\hline \multirow{2}{*}{ Staff Perceptions and influencing factors } & Confidence \\
\cline { 2 - 2 } & Fear of litigation \\
\cline { 2 - 2 } Patients factors and expectations & Interprofessional relations \\
\cline { 2 - 2 } & Psychosocial factors \\
\cline { 2 - 2 } Lack of standardised pathways and protocols & Pain \\
\cline { 2 - 2 } & Expectations \\
\cline { 2 - 2 } & Communication with Neurosurgery \\
\cline { 2 - 2 }
\end{tabular}

Table 5: Sub-themes of Challenges /Barriers.

\begin{tabular}{|c|c|}
\hline \multirow{2}{*}{ MRI scan and reporting } & Daily reserved MRI slot \\
\cline { 2 - 2 } & MRI reporting \\
\hline Staff education and training & GP and secondary care training and shared learning \\
\cline { 2 - 2 } & Competency framework \\
\hline Standardisation of pathways and protocols & Local and National Pathway \\
\hline
\end{tabular}

Table 6: Sub-themes of Solutions.

\section{Discussion}

In general the findings from this study reinforce the complexities of managing patients with suspected CES and a multi-faceted approach is required to optimise patient care. It was apparent during the data analysis that there are several challenges in the day-to-day management of patients with suspected CES, some of which could be improved with local agreement whilst some problems may require national 


\section{Journal of Orthopedics \& Bone Disorders}

agreement. Hussain, et al. proposes a national referral pathway to reduce inconsistencies in the management of CES [11].

\section{Staff Confidence}

In general, staff are confident in their knowledge of CES and clinical assessment to ensure accurate detection of those requiring escalation for MRI scan. This is not surprising as only experienced clinicians were invited to participate. There was however recognition that despite good clinical expertise, staff were not confident that clinical finding would correlate with the MRI. This is in line with findings of Hussain, et al., Balasurbramanian, et al. and Fairbank, et al. who demonstrate poor correlation of clinical assessment with MRI findings [11-13]. One clinician stated: "The majority of patients have normal scans despite symptoms suggestive of CES."

The general feeling is that clinicians accept this unpredictability and as a result there is a low threshold for MRI. The role of MRI has become increasingly important based on emerging evidence that early intervention can reduce disease progression and relapse rates [14]. A few clinicians described the difficulty in determining the significance of the "soft signs" or "grey areas" described in the subjective examination. Such descriptions relate to the overall challenges associated with this particular patient group and concludes that MRI is required for primary triage and confirmation of diagnosis [4,11].

Interestingly, very few clinicians stated they relied on intuitive judgement in diagnosing CES. This is reassuring as potentially fewer cases will be missed and would not be supported by existing guidelines [15]. However T\&O and ED clinicians acknowledge it is difficult to fully exclude intuition. Intuitive decision-making has been found in some cases to improve decisions and leads consistently to better performance than analytic deliberation [16]. Some clinicians suggested first impression is used more with regards to whether or not to contact Neurosurgery to discuss patient transfer for MRI scan during $00 \mathrm{H}$. It is arguable to what extent first impressions are representative of gut feelings. First impressions are considered perfectly rational and justifiable [16].

\section{Fear of Litigation}

The T\&O consultants are more aware of the significant impact of litigation and associated compensation figures. T\&O quoted settlement figures of $£ 500,000$ which reflects the clinicians' and the Health Boards' concern in managing CES. APPs and ED consultants acknowledge the threat of litigation but believe their final decision is based on patient safety. This is not to suggest that patient safety is considered any less by $T \& O$. T\&O clinicians have to manage all patients with suspected CES, not just those referred from ED, but also direct referrals from GPs' and other specialities within secondary care. There are daily pressures to ensure patients have a timely assessment, MRI and appropriate referral onwards. The responsibility ultimately lies with the Consultant oncall and therefore the threat of litigation may appear more significant within this clinical specialty [17]. Once a clinician has experienced litigation, it can have a detrimental impact on their clinical judgement and reasonable to assume, leads to over-treatment and over-investigation to prevent patient harm. It has been suggested that this form of defensive medicine creates barriers to innovation within the modern NHS [18].

Although the NHS is growing a culture of patient empowerment, there is still an acceptance and dependence on the clinician to make the final outcome [19]. It could be argued that similar problems exist amongst clinicians with reluctance to challenge the clinical decisions that are made, regardless of hierarchy. One ED consultant acknowledged that she wouldn't miss an obvious CES and that litigation usually precedes atypical presentations where there are other comorbidities and psychosocial factors. This was further supported by an APP who used the term "barn door case of CES" to describe patients with obvious signs including acute onset of severe LBP, bilateral leg pain and associated perianal, urinary and bowel symptoms. However it is well documented that there is no definitive subjective complaint or objective clinical findings that can confirm CES and therefore it is very difficult to know what constitutes as obvious signs of CES [4].

\section{Inter-Professional Relations}

The main concern raised from ED clinicians was the referral onto a considerably lesser-experienced medical staff, leading to unnecessary delays in patient management and diagnosis. Interestingly, during the NGT this was discussed in that the "referral down" to a junior colleague was not satisfactory, but is a valuable part of training and senior clinicians oversee final decisions.

APPs have similar concerns with regards to feedback from those they refer onwards and also experience difficulties getting referrals accepted, with variability being a factor. In view of changes in medical working patterns and busy schedules there is limited opportunity for effective feedback. Therefore enabling clinicians to improve their work performance by setting feedback-based goals will benefit their work in the long run [20].

One APP also highlighted concerns of poor inter professional respect: "It can be difficult convincing another 


\section{Journal of Orthopedics \& Bone Disorders}

clinician of your clinical concern of a patient they have not seen yet"

Variability of communication channels appears to be a concern and perhaps reflects the need for improved collaborative team working and standardisation of referral pathways in managing a patient with suspected CES.

In the author's place of work an APP has been autonomously managing patients with suspected CES in working hours (08:30-16:30), including perianal and bladder assessment and discussion with the Neurosurgical team. This single point of contact also improved the consistency in feedback. There was unanimous recognition that this has been a positive step forwards but sustainability, especially $\mathrm{OOH}$, is required. Although APPs are known to have an important role in the management of low back pain and CES, there does not appear to be any literature on the benefits of a frontline APP within ED autonomously managing a patient with potential CES. This perhaps merits more exploration to establish the benefits of consistent senior first point of contact on patient care and service delivery.

\section{Patient Factors and Expectations}

Psychosocial factors: Woods, et al. [5] and Greenhalgh, et al. [8] and have proposed comorbidities, medication and pain pose challenges to accurately detect CES [5,8]. The findings from this study identify chronic pain behaviour, anxiety and depression to influence decision-making. These views were mostly that of ED staff and APP's. There has been considerable research into chronic pain and the recognition that pain is not purely a physical sensation but has biological, psychological and emotional contributing factors. It is also rare to see a simple pain presentation [21]. An awareness of the psychosocial factors is not only favourable for clinical assessment but also as a predictor for post-surgical recovery. Confirmative diagnosis does not necessarily result in a successful surgical outcome [22].

Pain: High levels of pain and medications have been suggested to dilute the emerging symptoms of CES $[5,8]$. Pain was acknowledged to be more of a challenge within ED than by T\&O or APP clinicians, which likely reflects the clinical setting and patient acuity. However, one T\&O Consultant suggests "over-reliance on analgesia can be problematic and produce secondary side effects that further challenge clinical assessment."

One ED consultant expressed the challenge of conducting a complete assessment in a patient with high pain levels. Another colleague mentioned that most patients in severe pain are often affected by radiculopathy and not all patients with CES have high levels of pain.
Expectations: All clinical specialities mentioned the pressure experienced by patient expectations, often caused by social media, internet and from the preceding GP consultation. Interestingly, as much as $70 \%$ of litigation relates to real or perceived problems involving doctors' communication ultimately influencing patient expectations [23]. During the NGT there was a general consensus that GP consultation can have a significant impact on patient expectations leading to challenging conversations regarding the reason for referral to secondary care and escalation for MRI scan. Some clinicians feel ".....backed into a corner." Surprisingly, patients' unknown expectations were not discussed. For example, it is not unreasonable to assume some patients do not understand why they have been referred to secondary care and the clinical urgency associated. This supports the findings by Greenhalgh, et al. [8].

Lack of standardised pathways and protocols: There appears to be a general dissatisfaction with variability in the care provided to patients with suspected CES across the different clinical specialities. The dominant feature relates mostly to $\mathrm{OOH}$ presentations due to lack of MRI availability and ED consultants reinforce their frustration of $\mathrm{OOH}$ care. Some clinicians do not get sufficient exposure to patients with suspected CES that arguably will impact on their overall confidence and timely management. Ownership of patients during $\mathrm{OOH}$ due to poorly defined roles ultimately results in patient admission for senior decision-making and MRI scan. One T\&O Consultant stated:

"OOH attendances equates to an admission. There needs to be more thought into the patient pathway and options to avoid this often unnecessary step."

There was unanimous recognition of improved patient journey at the author's workplace when these patients are managed by an APP with specialist interest in CES. It was reassuring to find that several clinicians reflected on their past experience of managing CES and most of the concerns focused around the detrimental impact of inconsistent pathways. Repeated perianal assessment by different clinicians was highlighted by ED and T\&O to be unnecessary and unfavourable to patients [24].

The APPs within a T\&O clinic setting (excludes APP working in ED) highlighted that they do not conduct perianal assessment and referral onwards is based on subjective findings yet GPs' conduct full perianal assessment prior to referral to secondary care. This may reflect differences in competencies and previous training but nonetheless should be challenged to ensure patients have the most appropriate, least intrusive management. Suggestions of a working algorithm to enable consistent assessment were suggested to improve standards of care. 


\section{Journal of Orthopedics \& Bone Disorders}

\section{Access to MRI}

Access to MRI was a consistent challenge raised by all participants. There is general support from the Radiology department at the author's workplace, with praise of their efforts to arrange an MRI despite the daily pressures of urgent demand and staff shortages. The barriers focused mostly on lack of access to MRI $\mathrm{OOH}$ and the subsequent effects this has on patient care. This reflects the national challenges outlined by Germon, et al. [15] Concerns were raised about timescale of presenting symptoms and acceptable wait for MRI. A common solution identified was to create reserved daily MRI slot(s) solely for CES. Lengthy wait for MRI has been considered to negatively impact on patient care and inefficient scheduling has been recognised [25]. Optimising MRI scheduling could potentially reduce access time and enable more timely diagnosis for patients with CES. It could be argued though that in a time of rising NHS pressures we have to consider the safest, yet most realistic option to diagnose CES and perhaps there should be more emphasis on timescale of presentation in deciding acceptable wait for MRI.

\section{Interaction with Neurosurgery}

There was a general consensus from all disciplines of the challenges encountered when communicating with the tertiary Neurosurgical team. The main barriers that were highlighted include; lengthy timescale for discussion; variability in professional respect; variability in final outcome; and lack of definitive answer at first contact. One T\&O surgeon commented the wait to contact Neurosurgery can be up to thirty minutes, and even longer for the registrar to confirm the final decision made. Unfortunately, these problems are not solely related to $\mathrm{OOH}$ challenges, although there are more interactions with Neurosurgery $\mathrm{OOH}$ if urgent MRI is indicated. The local protocol advises an MRI scan locally within working hours and thereafter contacts Neurosurgical team for advice. There is clear acceptance of their professional role but concerns are raised regarding the acceptance and transfer of patients':

"Neurosurgery at times can fail to take responsibility for a patient and patients' often wait too long for transfer once CES has been confirmed on MRI."

There was however acknowledgment and appreciation that the neurosurgical service is "over stretched" resulting in "considerable delay and cross-wires in communication as a result." Communication with Neurosurgery does appear to be challenging. This therefore supports the need for revised national pathways to support more efficient ways of working [11].

\section{Staff Education and Training}

All clinical specialities proposed improving multidepartmental training to assist with the clinical assessment of patients with LBP and potential CES. Inclusion of the Neurosurgeons to facilitate learning was also suggested, and although would be extremely beneficial, may be difficult to implement.

\section{Competency Framework}

ED and T\&O suggested the development of a clinical assessment tool pan Ayrshire for use in primary, secondary and $\mathrm{OOH}$ services, to improve the management of CES. This would help to standardise the clinical assessment of CES. This suggestion correlates with proposals to quantify clinical assessment of CES using The Cauda Scale (TCS) [4]. An evidence-based algorithm would not only improve clinical assessment but also help to monitor deteriorating symptoms [26]. It is reasonable to suggest a nationally agreed assessment tool would be most beneficial to standardise objective assessment.

\section{Standardisation of Local and National Pathways}

The majority of clinicians suggested redesigning the local pathway within NHS Ayrshire and Arran due to significant inconsistencies. Admission in most cases is not desirable for patients, staff or the organisation. However, the principal responsibility of health care providers is to "do no harm" and the benefits of an intervention must out-weigh the risk [27]. Unfortunately, the perceptions of ED consultants is that there is often little thought given to patient admission and, as a result of unclear guidelines on timescale of preceding symptoms, admission is the easiest and fastest route to access urgent MRI. Consideration of patient deterioration is another factor and has been highlighted to reduce the threshold for admission. Todd concludes it is impossible to determine the rate at which CES will progress [4]. However, it could be argued that patient discharge could be supported if patients are provided with detailed information on deteriorating symptoms. Transfer to the tertiary centre for Neurosurgical assessment appears to be less than desirable. It was suggested that transfer can take "up to ten hours". Considering the guidelines suggest urgent decompression within twenty four to forty eight hours, this delay undoubtedly causes concern amongst clinicians especially if a patient has deteriorating symptoms [15]. This strengthens the argument for improved modes of communication and new processes to ensure timely assessment and surgery.

Despite suggestion for local improvements, the majority of clinicians involved in this study supported the development 


\section{Journal of Orthopedics \& Bone Disorders}

of a nationally agreed driven protocol and pathway. However there was also appreciation that the pressures experienced by clinicians in Ayrshire are mirrored elsewhere. Most of the clinicians appreciated that nationally driven guidelines and pathways would help to address some of the daily challenges outlined. This supports findings from Hussain, et al. to streamline patient care [11].

\section{Conclusion}

This study explored NHS clinical staff perceptions of managing a complex, highly litigious Neurosurgical emergency with often multi-departmental challenges. Collectively, the three clinical specialities provided a new insight into the challenges experienced and supports the desire for nationally driven improvements $[4,11,15]$.

Suggestions for local pathway redesign could be achievable with the biggest limitation being access to MRI during $00 \mathrm{H}$. 24-hour access to MRI would reduce the burden of $\mathrm{OOH}$ admissions, which ultimately has secondary implications on service delivery within working hours. Proposals for MRI scheduling could be considered but factors such as resource, staffing and unscheduled demand would need to be explored.

Clinicians have already recognised improvement in CES management attributed to the appointment of an APP with specialist interest. It would seem reasonable to ensure sustainability of this model of care and perhaps establish similar roles in other Health Boards. In addition, the APP could potentially act as a CES co-ordinator, with a key role in staff education, aiming to bridge the gap between primary, secondary and tertiary care.

\section{References}

1. Fraser S, Roberts L, Murphy E (2009) Cauda Equina Syndrome: A Literature Review of Its Definition and Clinical Presentation. Archives of Physical Medicine and Rehabilitation 90(11): 1964-1968.

2. Lavy C, James A, Wilson-Macdonald J, Fairbank J (2009) Cauda equina syndrome. BMJ 338: 881-884.

3. Gardner A, Gardner E, Morley T (2011) Cauda Equina syndrome: a review of the current clinical medico-legal position. European Spine J 20(5): 690-697.

4. Woods E, Greenhalgh S, Selfe J (2015) Cauda equina syndrome and the challenge of diagnosis for physiotherapists: A review. Physiotherapy Practice and Research 36(2): 81-86.

5. Hall R, Jones K (2018) The lived experience of Cauda
Equina Syndrome: a qualitative analysis. Spine 56 (4): 41-45.

6. Maguire P, Pitceathly C (2002) Managing the difficult consultation. Clinical Medicine 3(6): 532-537.

7. Greenhalgh S, Finucane L, Mercer C, Selfe J (2018) Assessment and management of cauda equina syndrome. Musculoskeletal Science and Practice 37: 69-74.

8. Mcmillan SS, King M, Tully MP (2016) How to use the nominal group and Delphi techniques. International journal of clinical pharmacy 38(3): 655-662.

9. Fincham JE (2008) Response rates and responsiveness for surveys, standards, and the Journal. American journal of pharmaceutical education 72(2): 43.

10. Balasubramanian K, Kalsi P, Greenough CG, Seetharam MPK (2010) Reliability of clinical assessment in diagnosing cauda equina syndrome. British Journal of Neurosurgery 24(4): 383-386.

11. Fairbank J, Hashimoto R, Dailey A, Patel AA, Dettori JR (2011) Does patient history and physical examination predict MRI proven cauda equina syndrome? Evidence Based Spine Care Journal 2(4): 27-33.

12. Miller DH (2004) Biomarkers and surrogate outcomes in neurodegenerative disease: lessons from multiple sclerosis. NeuroRx 1(2): 284-294.

13. Germon T, Ahuja S, Casey AT, Todd NV, Rai A (2015) British Association of Spine Surgeons standards of care for cauda equina syndrome. The Spine Journal 15(3): S2S4.

14. Wooley A, Kostopoulou O (2013) Clinical Intuition in Family Medicine: More than First Impressions. Annals of Family Medicine 11(1): 60-66.

15. Markham D (2003) Attitudes towards patients with a diagnosis of 'borderline personality disorder': Social rejection and dangerousness. Journal of mental health 12(6): 595-612.

16. Gorman $D$ (2015) On the barriers to significantinnovation in and reform of healthcare. Internal Medicine Journal 45(6): 597-599.

17. Thomson R, Bowling A, Moss F (2001) Engaging patients in decisions: a challenge to health care delivery and public health. BMJ Quality Safety 10(1): 1.

18. Renn RW, Fedor DB (2001) Development and field test of a feedback seeking, self-efficacy, and goal setting model of work performance. Journal of Management 27(5): 
563-583.

19. Eccleston C, Morley SJ, Williams ADC (2013) Psychological approaches to chronic pain management: evidence and challenges. British journal of anaesthesia 111(1): 59-63.

20. Hart RA, Cabalo A, Bess S, Akbarnia BA, Boachie-Adjei 0 , et al. (2013) Comparison of patient and surgeon perceptions of adverse events after adult spinal deformity surgery. Spine 38(9): 732-736.

21. Lateef $F$ (2011) Patient expectations and the paradigm shift of care in emergency medicine. J Emerg Trauma Shock 4(2): 163-167.
22. Gooding BW, Higgins MA, Calthorpe DA (2013) Does rectal examination has any value in the clinical diagnosis of cauda equina syndrome? British journal of neurosurgery 27(2): 156-159.

23. Wessman BV, Moriarity AK, Ametlli V, Kastan DJ (2014) Reducing barriers to timely MR imaging scheduling. Radiographics 34(7): 2064-2070.

24. Myers $\mathrm{T}$ (2006) The value of care algorithms. Pharmacotherapy: The Journal of Human Pharmacology and Drug Therapy 26(9): S181-S192.

25. Slawomirski L, Auraaen A, Klazinga NS (2017) The economics of patient safety. Paris. The Organisation for Economic Co-operation and Development. 